schliesslich löst man ihn in Alkohol und fällt mit Wasser. Er bildet braune kleine Nadeln, die bei $260-261^{\circ}$ schmelzen. Die Analyse ergab folgendes Resultat:

$$
\begin{array}{ll}
\text { Ber. für } \mathrm{C}_{13} \mathrm{H}_{10} \mathrm{~N}_{2} \mathrm{O}_{4} & \text { Gefunden } \\
\mathrm{N} \quad 10.22 & 9.95 \mathrm{pCt} .
\end{array}
$$

Die Verbindung ist etwas in heissem Wasser löslich, gut in Alkohol, sehr leicht in Aceton, schwierig in Benzol, Chloroform und Ligroïn.

584. Nicolaus Zelinsky und Marc Feldmann: Ueber symmetrisches Diphenyltrimethylencyanid und symmetrische Diphenylglutarsäure.

(Eingegangen am 13. November.)

Es ist kürzlich ${ }^{1}$ ) von dem Einen von uns bewiesen, dass die symmetrische Dimethylglutarsäure in zwei isomeren Modificationen zix existiren vermag. Indem wir zur Erledigung der Frage über die Isomerie solcher gesättigten Verbindungen schritten, die auf Grund der Structurtheorie nur in einer Modification auftreten dürfen, wurden wir ganz natürlich zu der symmetrischen Diphenylglutarsäure geführt, die uns synthetisch darzustellen gelang.

Das Hauptziel dieser Abhandlung war, zu untersuchen, ob die Existenz von zwei isomeren symmetrischen Diphenylglutarsäuren möglich ist. Die von uns erhaltene Diphenylglutarsäure enthält, ihrer Synthese aus Methylenjodid and Benzylcyanid nach, zwei asymmetrische Kohlenstoffatome und sollte demgemäss vom Standpunkte der van't Hoff-Le Bel'schen Theorie wenigstens in zwei isomeren Modificationen erhalten werden. Bei der vorliegenden Untersuchung verwandten wir um so grössere Aufmerksamkeit, als es uns, auf Grund einiger theoretischen Ueberlegungen, die wir jetzt noch nicht darthun wollen, sehr.wahrscheinlich war, dass, abgesehen von der vollständigen Analogie der Structur der symmetrischen Dimethylglutarsäuren und der Diphenylglutarsäure, die letztere gemäss ihrer Synthese aus Benzylcyanid und Methylenjodid doch nur in einer Modification existien wird. Die weitere Aufklärung dieser Frage wird nächstens von uus untersucht; einstweilen erlauben wir uns, die folgenden bis jetzt erhaltenen experimentellen Ergebnisse mitzutheilen.

1) Journal d. Russ. chem. Gesellsch. XXI, 389. Siehe auch diese Berichte XXII, 2823. 
$\alpha$ - $\alpha$-Diphenyltrimethylencyanid.

Zur Darstellung des $\alpha$ - $\alpha$-Diphenyltrimethylencyanids liessen wir Benzylcyanid, Methylenjodid und trockenes Natron, d. i. unter Anwendung von Victor Meyer's »Natronmethode» 1), aufeinander wirken. $23.4 \mathrm{~g}$ Benzylcyanid wurden mit $26.8 \mathrm{~g}$ Methylenjodid gemischt und zur erhaltenen Mischung in kleinen Mengen $8 \mathrm{~g}$ pulveriges geschmolzenes Aetznatron zugefügt. Die Mengen der angewandten Substanzen entsprachen der Gleichung:

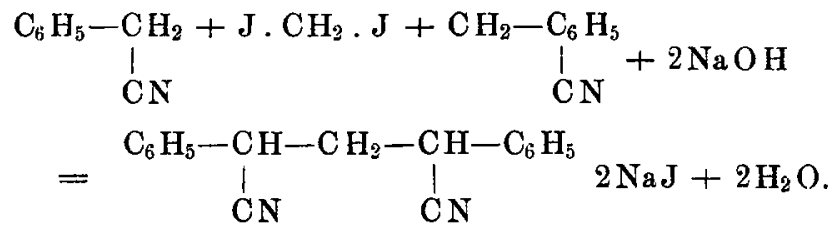

Der Kolben mit der erwähnten Mischung warde mit einem aufrecht stehenden Kühler in Verbindung gebracht und zur Einleitung der Reaction mit einer blauen Flamme erbitzt. Sobald die stürmisch verlaufende Reaction eintritt, muss die Flamme entfernt werden. Lässt sie nach, so wird der Kolben noch ein wenig erhitzt und somit das Ende der Einwirkung erzielt. Innerhalb 10-15 Minuten hat sich derart im Kolben eine ansehnliche Menge von Jodnatrium gebildet, das von einer schweren, dunklen und wenig beweglichen öligen Schicht bedeckt ist. Das ölige Product wird abgegossen, Jodkalium mit Aether gewaschen, die ätherische Lösung zum Product zugegeben und nach dem Abdestilliren des Aethers das Oel mit Wasserdämpfen destillirt, um nicht angegriffenes Methylenjodid und Benzylcyanid zu isoliren. Das mit Wasserdämpfen nicht übergebende Oel wurde vom Wasser getrennt und in heissem Alkohol gelöst.

Aus der alkoholischen Lösung schieden sich beim Abdampfen des Alkobols an der Luft sehr schöne, gut ausgebildete Krystalle ab, die abgepresst und aus Alkohol umkrystallisirt wurden.

Die nähere Untersuchung zeigte, dass diese $\mathrm{Krystal}^{\mathrm{l}} \mathrm{e}$ nichts anderes, als das von uns gesuchte Nitril der Diphenylglutarsäure ist. Die Untersuchung der Krystallform von dieser Substanz wurde auf unsere Bitte von Herrn Privatdocent Prendel ausgeführt, der uns Folgendes mittheilt:

»Die Krystalle der untersuchten Substanz besitzen eine plattenartige Gestalt und sind zu fächerförmigen Aggregaten und Stäben angeordnet. Ihre Grösse beträgt von $0.5-1.5 \mathrm{~mm}$. Bei der Untersuchung unter den Mikroskope im parallelpolarisirten Lichte erwiesen sie sich doppelbrechend; sie besitzen eine starke Doppelbrechung,

1) Ann. Chem. Pharm. 250, 123. 
indem sie in den feinsten Schuppen die Interferenzfarben der ersten Ordnung geben. Auf allen Schnitten tritt bei ihnen eine gerade Auslöschung statt, sie gehören demnach dem rhombischen System an. Alle stellen sie Combinationen von einem Prisma, einem Brachydoma und einem Macropinakoïd vor, siehe die beiliegende Figur. Beim Zerquetschen mit dem Deckgläschen geben sie eine Reibe von Spalten, die dem Brachypinakoïd parallel sind; man darf also mit Recht annehmen, dass die vollkommenste Spaltbarkeit

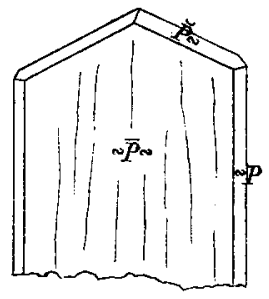
dieser Ebene parallel gerichtet ist. Bei zwei und zwanzig Krystallen wurde der Winkel der Brachydoma gemessen (im brachydiagonalen Schnitte unter dem Mikroskop) und das Mittel dieser Messungen gab 128 . Da aber hier keine andere Domen gefunden waren, so berechnet sich das Verhältniss der Parameter, indem wir diese Dome für die Hauptdome annehmen, zu:

$$
\overline{\mathrm{b}}: \dot{\mathrm{c}}=1: 0.4877 \text {. }
$$

Das Verhältniss $\breve{a}: \bar{b}$ konnte nicht berechnet werden, weil kein einziger Krystall mit einer guten Ausbildung der Prismenflächen vorhanden war.\&

Die Verbrennungen dieser Substanz gaben folgende Resultate:

I. $0.1589 \mathrm{~g}$ Substanz mit Kupferoxyd verbrannt gaben $0.4863 \mathrm{~g}$ Kohlensāure und $0.0838 \mathrm{~g}$ Wasser.

II. $0.1255 \mathrm{~g}$ Substanz mit Kupferoxyd verbrannt gaben $0.3820 \mathrm{~g}$ Kohlensäure und $0.0658 \mathrm{~g}$ Wasser.

III. $0.1532 \mathrm{~g}$ Substanz mit Kupferoxyd verbrannt gaben bei Bar. von $0^{0}$ $=762 \mathrm{~mm}$ und bei $t^{0}=1 S^{0}$ ein Volum von $15.1 \mathrm{cem}$ feuchten Stickstoffs.

\begin{tabular}{|c|c|c|c|c|}
\hline & I. & $\begin{array}{l}\text { Versuch } \\
\text { II. }\end{array}$ & III. & Ber. für $\mathrm{C}_{\mathrm{tz}} \mathrm{H}_{14} \mathrm{~N}_{2}$ \\
\hline C & 83.45 & 83.01 & - & 82.93 pCt. \\
\hline $\mathbf{H}$ & 5.87 & 5.82 & - & 5.69 \\
\hline $\mathbf{N}$ & - & - & 11.41 & 11.38 \\
\hline
\end{tabular}

Zur Ermittelung des Moleculargewichtes dieses Körpers benutzten wir Raoult's Methode ${ }^{1}$ ).

I. Versuch in Essigsãure. Gew. der Essigsãure - 21.2416; Gew. der Substanz $=0.0694 \mathrm{~g}$; die Erniederung des Gefrierpunktes $=0.055^{\circ}$.

II. Versnch in Benzol. Gew. des Benzols $=15.660 \mathrm{~g}$; Gew. der Substanz $=0.1473 \mathrm{~g}$; Erniederung des Gefrierpunktes $=0.185^{\circ}$.

1) Unter Anwendung des Beckmann'schen, in 1/100 Grade getheilten Thermometers. Zeítschrift f. physik. Chem. II, 644 . 


\begin{tabular}{ccc}
\multicolumn{2}{c}{ Versuch } & Ber. für $\mathrm{C}_{17} \mathrm{~B}_{14} \mathrm{~N}_{2}$ \\
I. & II. & 246
\end{tabular}

Wir hatten es hiernach in der That mit dem Nitril der symmetrischen Diphenylglutarsäure zu thun, denen beiden auf Grund ibrer von uns ausgeführten synthetischen Darstellung folgende Formeln zuzuschreiben sind:

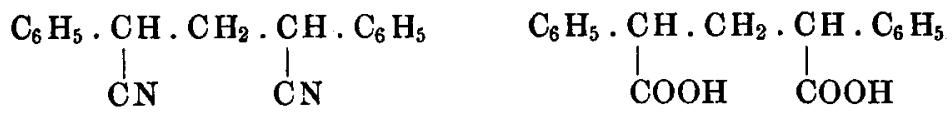

Der Schmelzpunkt des Nitrils liegt bei $70-71^{\circ}$. Die Ausbente ist genügend: aus $23.4 \mathrm{~g}$ Benzylcyanid und den entsprechenden Quantitäten von Methylenjodid und Natron werden bei der ersten Krystallisation $8 \mathrm{~g}$ reinen Nitrils erhalten, während die Mutterlauge noch eine verhältnissmässig ansehnliche Menge enthält.

\section{Symmetrische Diphenylglutarsäure.}

Das Product der Einwirkung von Methylenjodid auf Benzylcyanid, das nach dem Abdestilliren mit Wasserdämpfen von dem nicht angegriffenen Methylenjodid und Benzylcyanid im Kolben blieb, wurde in wenig Alkohol gelöst und zu einer starken wässerigen Lösung von Aetzkali zugegossen. Die Verseifung des Nitrils verläuft schnell und ist nach 6-8 stündigem Erwärmen völlig beendet. Beim Ansäuern der Lösung mit Salzsäure fällt eine organische Säure, als ein sehr schweres und dichtes Oel nieder, das nach kurzer Zeit erstarrt. Die Säure ist sehr schwer löslich, sogar im heissen Wasser, aus dem sie sich aber umkrystallisiren lässt. Beim Erkalten der wässerigen Lösung scheidet sich die symmetrische Diphenylglutarsäure in kleinen Nadeln aus, die sich knospenartig gruppiren. Ihr Schmelzpunkt liegt bei $164^{\circ}$.

Bei der Analyse wurden folgende Resultate erhalten:

I. $0.1465 \mathrm{~g}$ Substanz mit Kupferoxyd verbrannt gaben $0.3867 \mathrm{~g}$ Kohlensäure und $0.0775 \mathrm{~g}$ Wasser.

II. $0.1142 \mathrm{~g}$ Substanz mit Kupferoxyd verbrannt gaben $0.3010 \mathrm{~g}$ Kohlensăure und $0.0613 \mathrm{~g}$ Wasser.

\begin{tabular}{cccc} 
& \multicolumn{2}{c}{ Versuch } & Theorie \\
& I. & II. & für $\mathrm{C}_{17} \mathrm{H}_{16} \mathrm{O}_{4}$ \\
C & 71.98 & 71.87 & $71.83 \mathrm{pCt}$. \\
H & 5.87 & 5.96 & 5.63
\end{tabular}

Aus der Säure wurde durch Fällen des Ammoniumsalzes mit Silbernitrat ein Silbersalz erhalten und analysirt:

$0.1530 \mathrm{~g}$ Silbersalz gaben beim Glühen $0.0647 \mathrm{~g}$ Silber.

Gefunden

Ag $\quad 43.09$
Ber. für $\mathrm{C}_{17} \mathrm{~K}_{14} \mathrm{Ag}_{2} \mathrm{O}_{4}$

43.37 pCt. 
Nachdem sich auf solche Weise die in heissem Wasser schwer lösliche Sãure als die von uns gesuchte symmetrische Diphenylglutarsāure erwiesen hatte, wandten wir uns natürlich zu der wässerigen Lösung und hofften darin eine löslichere isomere Säure $z u$ finden. Durch vielfaches Extrahiren dieser Lösung gelang es, ganz geringe Mengen einer organischen Säure in Form von kleinen Platten zu erhalten, deren Schmelzpunkt bei $72-74^{\circ}$ liegt. Sehr wahrseheinlich liegt die Phenylessigsäure vor, entstanden durch Verseifung kleiner Mengen Benzylcyanids, welches bei der Destillation mit Wasserdämpfen nicht völlig abgetrieben war.

Es ist also durch das vorläufige Studium der symmetrischen Diphenylglutarsäure einstweilen die Existenz von nur einer Modification constatirt. Die nächste Untersuchung muss die Frage entscheiden, in wie weit die von uns gemachte Voraussetzung betreffs der Existenz nur einer symmetrischen Diphenylglutarsäure richtig ist. Diese Frage in Verbindung mit der zweifachen Isomerie der symmetrisch substituirten Glutarsäuren, die synthetisch aus $x$-Cyanpropionsäureester und Methylenjodid dargestellt werden, wird jetzt im hiesigen Laboratorium von Einem von uns zusammen mit einigen seiner Mitarbeiter untersucht.

Was das beschriebene Nitril betrifft, so scheint es als sehr interessantes Material für weitere synthetische Arbeiten dienen zu können. Wir denken durch Reduction daraus das symmetrische Diphenylpentamethylendiamin, und aus diesem Diphenylpiperidin folgender Gleichung gemäss darzustellen:

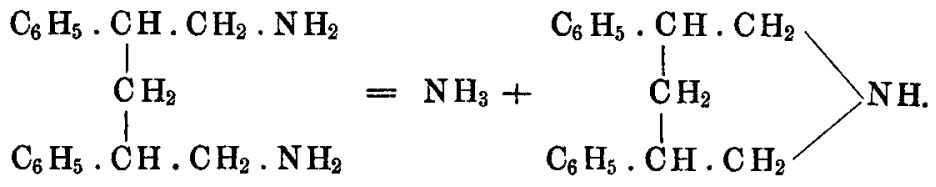

Der Versuch der Umwandlung des Nitrils der Diphenylglutarsäure in Diphenylpiperidin wird zum Gegenstande des nächsten Studiums für Einen von uns ( für M. Feldmann) sein.

Odessa, im November 1889. Organische Abtheilung des chem. Laboratoriums der Universität. 